\title{
Triakonta BB100: Dynamic Systemization Meets Big Bamboo
}

\author{
"Less is more." (Mies van der Rohe, New York Herald Tribune, June 28, 1959). \\ Less is core. (David Wharton, the-neighbourhood.com/thinking, Dec. 12, 2014) \\ "How much does your building weigh? (Buckminster Fuller, 1978) \\ "How much does your building impact? (Jack Elliott, 2015)
}

\section{INTRODUCTION}

Bamboo has long been used as a vernacular building material in tropical regions all around the world. Methods of construction have typically involved ad hoc, inexact processes of in situ cutting, drilling, notching and lashing, relying on local building cultures and knowledge. However, in a globalizing economy, this traditional means of building has become associated with poverty and/or cultural nostalgia. Bamboo construction is not widely accepted as a viable, modern means to making buildings in tropical markets, despite its many environmental benefits. Bamboo is a rapidly renewable material that can grow in exhausted soils, phytoremediate brownfield sites, control erosion, and sequester carbon. Using bamboo reduces harvesting pressures on much slower-growing local forests. Additionally, not only are the bamboo fibers strong and light, but their configuration as a hollow tube is extremely efficient for structural applications. Some recent commercial attempts have been made to use bamboo for architectural structures but they are either prohibitively expensive because of the lack of standardization and labor-intensive techniques or the fibers are reconstituted into dimensional lumber, no longer recognizable as bamboo.

The Triakonta BB100 structural system was developed at Cornell University to overcome these problems. It is designed to offer a lower cost alternative to building with bamboo than with other bamboo building systems available in tropical markets. It is also designed to provide great formal variety using a very limited set of components, rather than customizing every component for each new project. This substantially reduces production costs. Finally, it is designed for reversibility, both in assembly as well as component construction. This allows for greater responsiveness and flexibility for changing functions or locations.

The Triakonta BB100 system responds to real building needs in tropical contexts, but is not intended for widespread use in mid-latitudes, both North and South. However, it does provide some important directives for contemporary practice in these non-tropical contexts. Whether using bamboo or not, lowering the embodied energy of buildings is becoming more important as operational energies of buildings drop. Designers must become more aware of the carbon footprint of the building materials they specify. They also must
JACK ELLIOTT

Cornell University 
become more aware of the end of life issues associated with buildings. Typically, they are demolished, losing most of their material's potential for reuse. Designing for disassembly ensures that at its end-of-life, the building is not destroyed but is reconfigured or dismantled. Designing for reversibility ensures that all of the component materials can be separated for recycling or repurposing. Building can be reconsidered as a new way of storing materials.

\section{BAMBOO BACKGROUND}

The species of bamboo used for the Triakonta BB100 system is Guadua angustofolia, the world's largest bamboo, native to South America and widely cultivated in Colombia. This rapid-growth building material has been used for centuries but since European settlement has come to be regarded as a substandard building material because it is a cheap, accessible material commonly used for improvised housing ${ }^{1}$. However, recent uses of bamboo in high-end homes and high profile projects have brought renewed attention to the beauty, strength and elegance of this humble building material. The most emblematic example was the Zeri pavilion made of guadua, designed by Colombian architect Simón Velez for the 2000 Hannover Expo ${ }^{2}$.It has also been used increasingly in residential and commercial applications in Colombia. In 2010, guadua was included for the first time in the Colombian building codes, allowing for its use in residential one and two story buildings, an important step for increasing the credibility of Guadua as a building material ${ }^{3}$.

Bamboo is a grass, and this particular species grows up to 8 inches a day and reaches its full height in six months. The culm itself takes 4 to 5 years to mature into a structurally sound element. It can grow to a height of $100 \mathrm{feet}$, with a diameter up to 8 inches. Its compression strength fluctuates between $5000 \mathrm{Psi}$ and to over $7000 \mathrm{Psi}^{4}$, comparable to white oak. However, unlike trees, when the bamboo culm is harvested, it does not kill the plant. The well-established root system will send up another stalk in the next growing season to repeat the harvesting cycle.

The best time to cut bamboo is before sunrise just after the dry season has started. This time and season ensure the minimum amounts of carbohydrates and moisture are present in the stalks ${ }^{5}$. After being cut close to the ground, the stalks are left standing intact for four weeks, with the cut end placed on a stone to allow the parenchyma cells to continue to digest any reserves of starch in the plant. This process is known as post-harvest transpiration and it is done primarily done to improve the culm's resistance to insects, but the dryer, lighter stalks are easier to transport and will finish drying faster.

The culms are then transported to a mill, where they are given a chemical treatment to preserve and protect the bamboo. The treatments depend on their uses: exterior applications use fixing preservatives such as ACQ while interior applications typically use boron salts. These treatments are done through a leaching process, where the culms are drilled longitudinally and wholly submersed in treatment tanks for 3-4 weeks. After treatment, the culms are air-dried out of the sun, usually in horizontal stacks. The poles are rotated end for end every two weeks over the drying period. This takes 6-12 weeks but is a necessary step to prevent cracking of the stalks. Afterwards, the treated, dried culms are cut into 20 -foot lengths and are sorted according to their two end diameter sizes. The largest pieces are $6 "-7 "$, ranging down to $2 "-3 "$.

\section{THE TRIAKONTA BB100 SYSTEM}

The Triakonta system is named after the rhombic triacontahedron that it uses as a structural node. Rather than use spheres that are custom-drilled for each application, a regular uniform polyhedron with flat faces was sought to provide a better bearing surface for the ends of the struts. Examples of polyhedral nodes from industry usually include Archimedian solids such as the truncated cubes, like the cuboctahedron, or the truncated octahedron. 


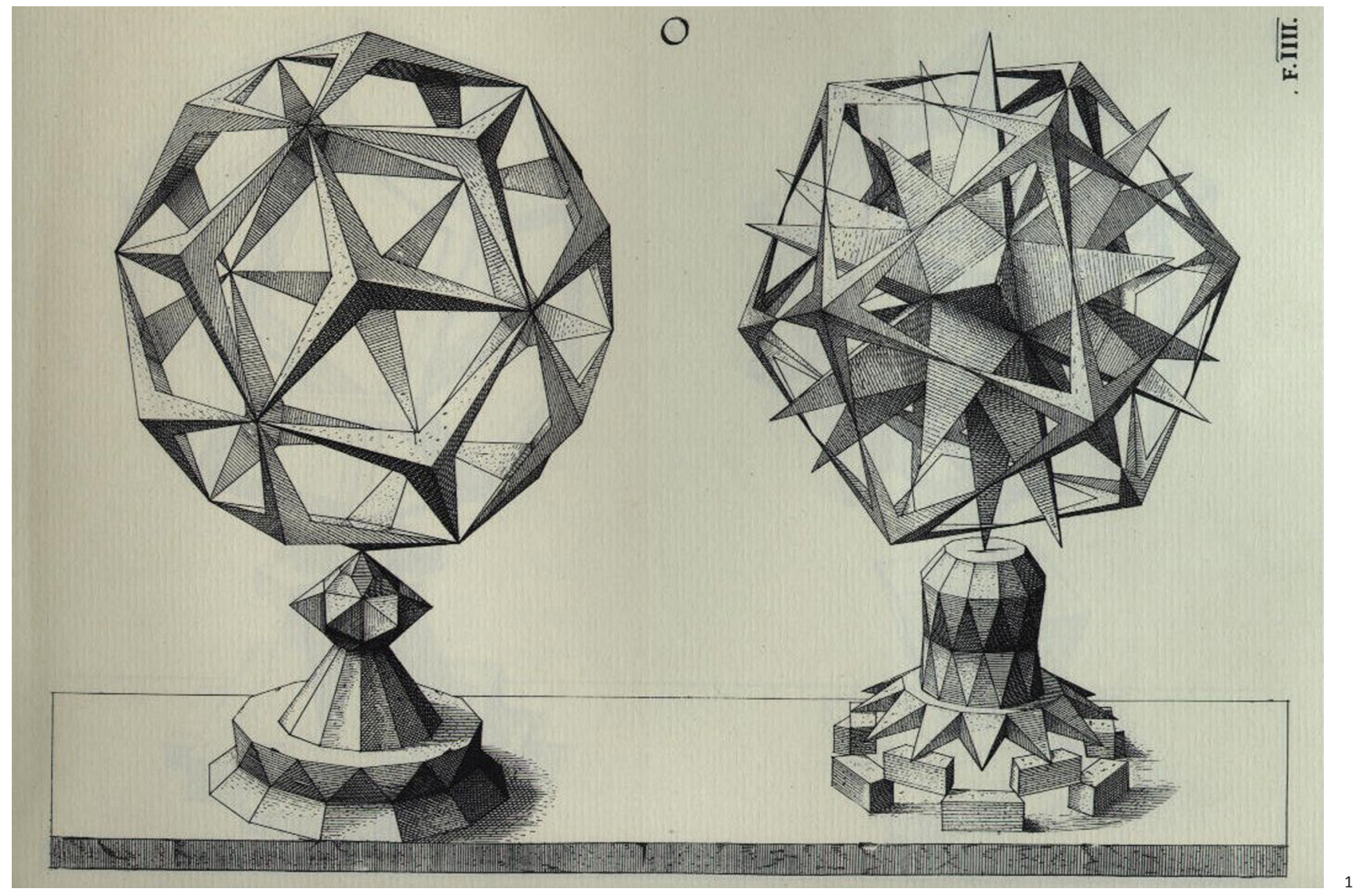

However, these have limited internal angular relationships. Instead, a 30-sided convex polyhedron known as a rhombic triacontahedron, was selected, hence the product name "Triakonta". The "BB" refers to "bamboo" and the "100" refers to the metric measurement across the opposite flats of the node. This polyhedron was first discovered and depicted by Jamnitzer in 1568 (Fig. 1), although it is often attributed to Kepler, whose mathematical description appeared 43 years later. This shape allows for orthogonal structures with right angular relationships, as well as triangular, pentagonal, and hexagonal arrangements.

In addition to this geometric complexity, the Triakonta system is unique among geodesic structural systems for its use of Guadua culms for the struts of this geodesic system. Typical geodesic systems rely on metal tubing, precise and uniform but with little character. The Triakonta system exploits the hollow configuration and familiar look of the natural material while allowing for its irregularities of dimension and form within a standardized set of building components. The structures have a "natural" look but have very tight tolerances. These are shipped to the jobsite where they are placed in jigs to ensure they are cut to exact lengths and drilled in precise locations for the attachment of end anchors.

The anchors are made from $0.625^{\prime \prime} \times 16^{\prime \prime}$ stainless steel bolts inserted through a hole drilled in one end of a two inch length of $0.25^{\prime \prime}-2^{\prime \prime} \times 6$ " aluminum rectangular hollow section (R.H.S.). The bolt is free to rotate and move along its axis. Perpendicular to the bolt on the other end of the R.H.S., the sidewalls are drilled to accommodate a 1.25 " diameter by 6" aluminum pin, which fixes the assembly to the side walls of the bamboo. A concrete cone is placed over the bolt to allow a number of struts to converge on a single node without interference. These cones are made of Ultra-High Performance Concrete to provide the ductility and tensile strength necessary, cast in 3d-printed molds. A stainless steel dowel pin is forcefit into a tight tolerance hole that is located on the exposed end of the bolt. A broached

Figure 1: Triacontahedron on the left, (Intaglio print: Jamnitzer, Perspectiva Corporum Regularium, Jamnitzer, 1568): Plate F IIII. 


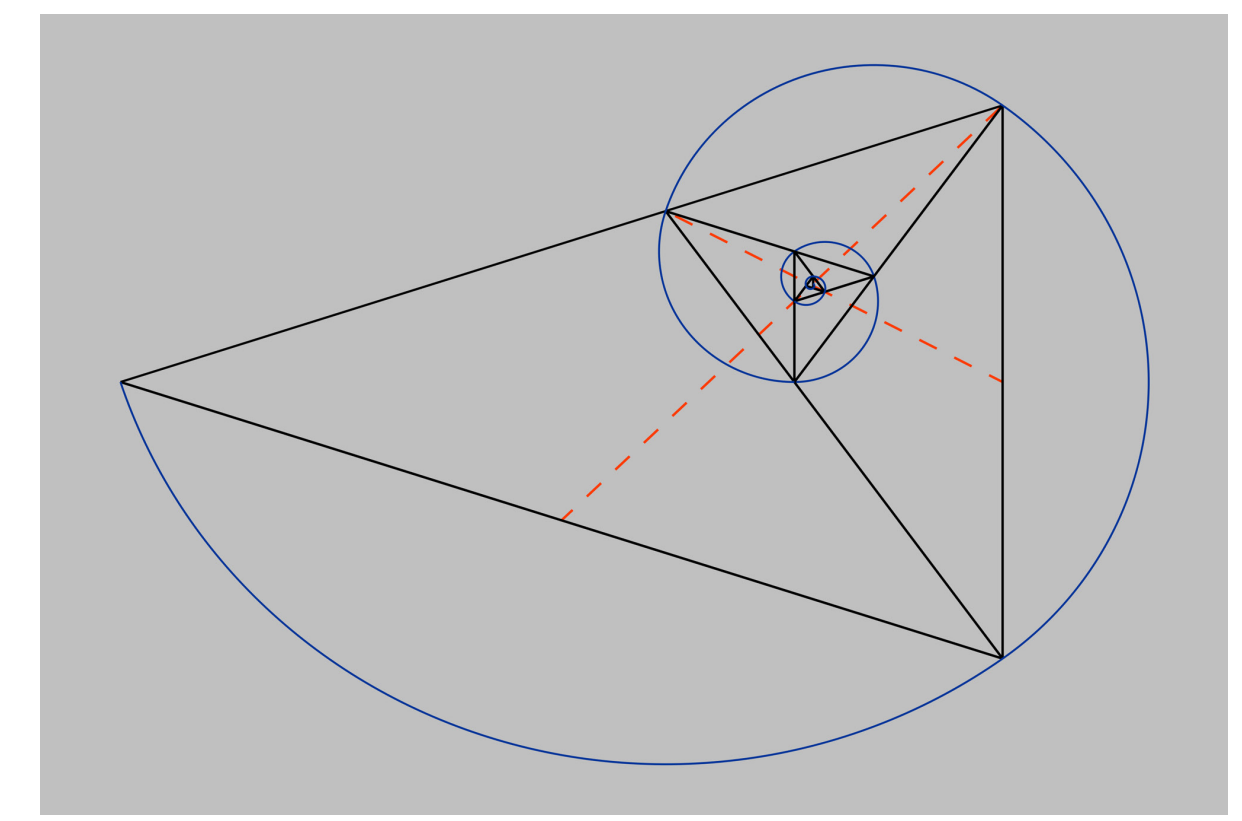

stainless steel collar fits over the bolt and engages with the pin. The collar is drilled on the outside to accommodate a pin wrench which is used to rotate the bolt in or out of the node. This single specialized tool is used for the entire assembly/disassembly process while rendering the structure tamper-proof.

Connecting the rhombic triacontahedral nodes automatically generate dynamic/golden/ divine relations between the component lengths, as referenced in the title of this paper. The term "dynamic" is used in the geometric sense as defined by Jay Hambidge in his book "The Elements of Dynamic Symmetry"6 referring to uniform growth often found in nature. His use of the term 'symmetry' is understood as the Euclidian concept of commensurability. His principle of proportioning was derived from the study of Greek building and artifacts where "root rectangles" and their diagonals produced a system of geometric relations.

In the case of the Triakonta system, these relations are based on $\sqrt{ } 5$ rectangles, which imbed the Golden ratio. Connecting three rhombic triacontahedrons with straight line segments normal to their faces produces triangles that are either equilateral or isosceles. The isosceles are of two types: narrow or wide (Fig. 2). In either case, the lengths of the sides of the isosceles triangles are related to their bases by the Golden ratio or its inverse. Thus, the rhombic triacontahedron produces a three-dimensional system using only three different lengths: short, medium, and long, each greater than the preceding one by a factor of $1.618 \ldots$. The result is great configurational variety with few standardized components. Additionally, the system is designed for disassembly, so that the building can be modified to accommodate changing conditions or at the end of life, can be completely dismantled and re-configured for a new site or set of conditions.

A working prototype of the Triakonta BB100 system is about to be erected this summer and field-tested in the Dominican Republic in the form of a simple gable structure (Fig. 3). It is triangulated on the outside to allow for a large free-space inside to serve as a classroom or meeting space at the Puntacana Ecological Foundation, a non-profit arm of the Puntacana Resorts and Club.

For the past 19 years, the mission of the Foundation has been to protect and restore the

Figure 2: Golden triangles in Fibonacci spiral (Image: Wikimedia Commons) natural resources of the Punta Cana region and contribute to the sustainable development of the Dominican Republic. For the last four years, they have served as a local partner for 


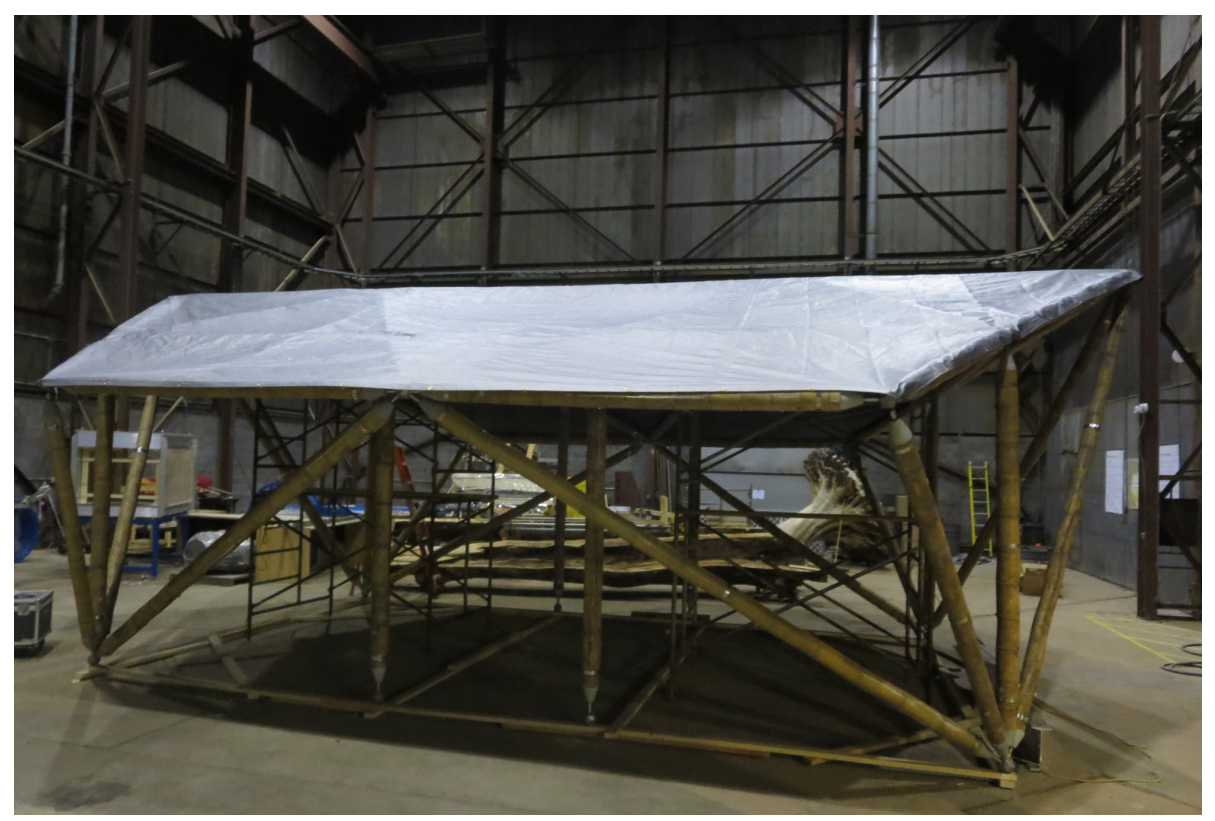

Cornell University's Triakonta research project. In addition to the system's inherent sustainability, its triangularity, low weight, and high strength make it suitable for this region's seismic activity and hurricane winds. If technically and culturally successful, the Triakonta system could form the basis of new local industry with vocational training for local contractors and even a new agro-forestry initiative sponsored by the Foundation. In this way, architecture could possibly play a role in reducing deforestation pressures while providing new safer, greener solutions to habitation in tropical and subtropical regions of the world. But what about the non-tropical places?

\section{GATHERINGS FROM GUADUA: EMBODIED ENERGY}

While this research is geared for projects in the developing world, the Big Bamboo project has some important suggestions for the developed world as well. One of the most important of these has to do with embodied energy, the amount of energy that is required to convert a raw material into a finished product delivered to the building site for its final disposal. As humankind starts to understand the impacts the built environment is having on the biophysical world, designers and architects need to re-think standard practices. On the issue of climate change, much work is being done on reducing the carbon footprint of buildings but mostly from an operations point of view. However, embodied energy is also an important factor for building impact, but is more difficult to quantify.

Certain researchers count the upstream energy consuming processes of a given product or building, while others consider the energy expended at every stage of the life cycle. ${ }^{7}$ Despite these inconsistencies and lack of a universal framework to calculate embodied energy, the current consensus is expressed by Manesh Dixit, a prominent American scholar on building energy assessment, who states that current research has "found that embodied energy accounts for a significant proportion of total life cycle energy." ${ }^{8}$ He cites two Australian studies that have demonstrated that embodied energy costs could be equivalent to a range of 15 to 50 years of operational energy $\operatorname{costs}^{9}$ (Fig. 4). Since the average age of a commercial building in the U.S. is 41.7 years ${ }^{10}$, if a building is a high performance building, more energy is embodied than consumed over its entire lifecycle. However, embodied energies of materials are just now being considered in green design protocols. The Living Building Challenge 3.0 in the "Materials" section under "Embodied Carbon Footprint", although not all energy
Figure 3: Triakonta BB 100 structure fully assembled, Cornell University, 2015 (Photo: Jack Elliott) 


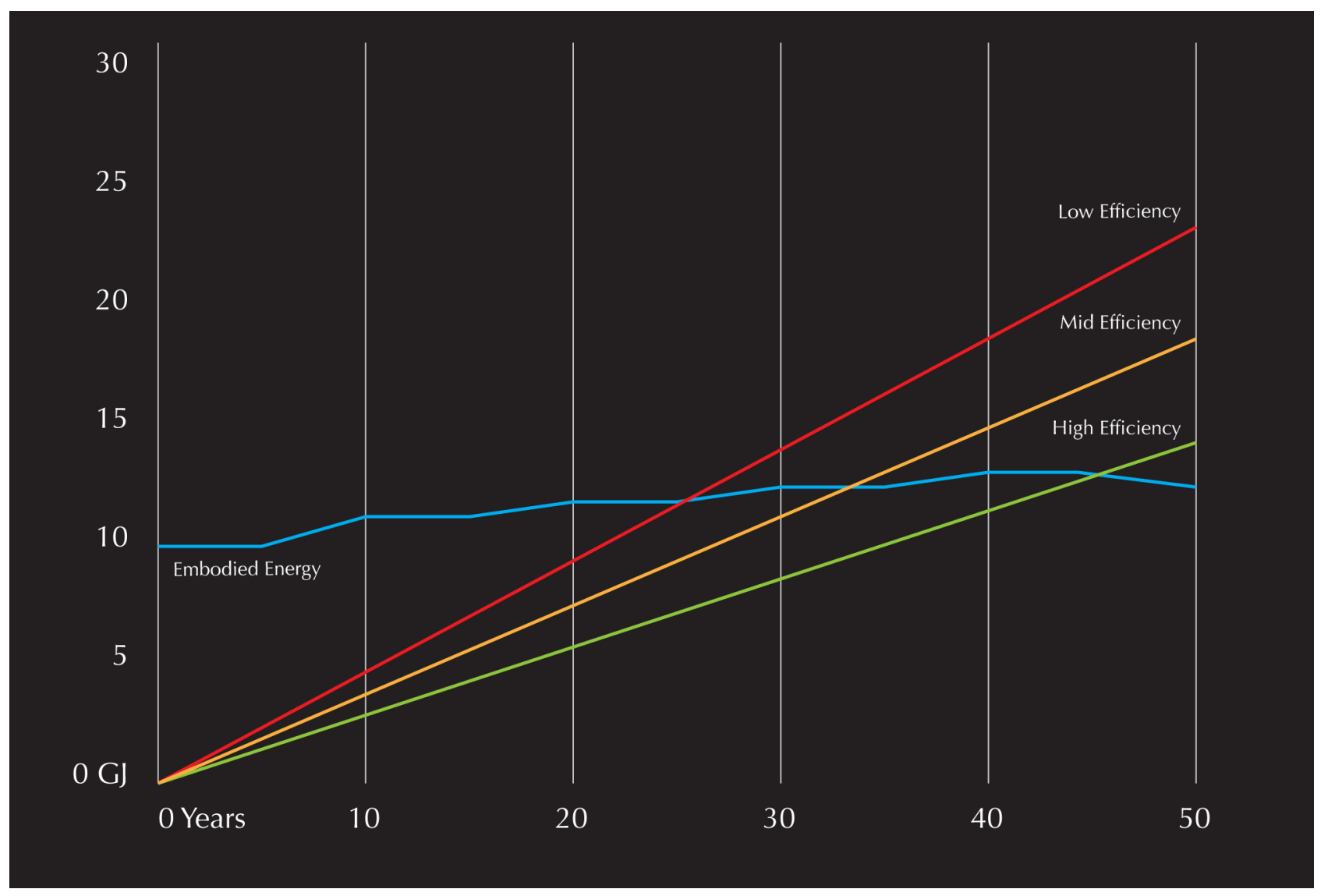

\section{ENDNOTES}

1. Marcelo Villegas. New bamboo: Architecture and design (Bogota: Villegas Editores, 2003): 34.

2. Alexander von Vegesack, Mateo Kries. Grow your own house: Simon Velez and bamboo architecture (Weil am Rhein, Germany: Vitra Design Museum, 2000): 14-49.

3. Ximena Londoño. "El bambú en colombia". Biotecnología Vegetal, 11 no.3 (2011): 147.

4. Oscar Hildago López. Bamboo: Gift of the Gods, (Bogota, Colombia: The Author, 2003). 88-90.

5. Stéphane Schröder, "When and How to Harvest Bamboo", guaduabamboo.com, Nov. 15, 2012, (http://www.guaduabamboo.com/cultivation).

6. Jay Hombidge, The Elements of Dynamic Symmetry, (Mineola, NY: Courier Corp., 1926).

Figure 4: Cumulative Energies of a Building (Graph: Adams, Connor and Ochsendorf, MIT OpenCourseWare) generates carbon. Renewable-energy-created cars may have a small carbon footprint but have large amounts of embodied energy. All energy is worth conserving, renewable or not. LEED v4 has a new credit called the "Building life-cycle impact reduction" but the term "embodied" is not included in the description.

This dynamic does not just apply to buildings. A recent article in Green Car Reports is entitled "As Gas Mileage Rises, Energy To Make Cars Gets More Important"11. Volkswagen completed a study in 2014 on its Golf 1.6 TDI where it found that the embodied energy of the car was $22 \%$ of its total carbon footprint over its lifetime, compared to $6 \%$ in 2000 .

\section{TEACHINGS FROM TRIAKONTA: DESIGN FOR DISASSEMBLY}

Another important concept that is often overlooked in the design and construction of the built environment is the end-of-life impacts of the intervention. Buildings are "demolished", "destroyed", "wrecked" by wrecking balls, "bulldozed" by bulldozers where all of the potential for re-use of the materials is lost. Building demolition wastes make up almost $50 \%$ of U.S. construction and demolition waste totals, which were estimated to be 136 million tons in $1996^{12}$. Not only is this a massive amount of material, it requires even more material to be extracted to replace that which is lost in the built environment. All of the methods we use to resupply these lost materials have substantial carbon footprints embodied with them. This planet cannot continue to be an unlimited source as well as sink. We need a new plan.

Imagine a scenario where instead of "demolish" or "destroy", words such as "dismantle" or "deconstruct" or "disassemble" where used to describe the end-of-life of a building. What are the implications of this? This means that the building would not rely on irreversible processes in its fabrication. No curing, no setting, no drying. Only reversible processes would be used. Screwing, bolting, clipping, fitting. No wet processes, only dry. The building becomes a new way to store material, recyclable or otherwise. 


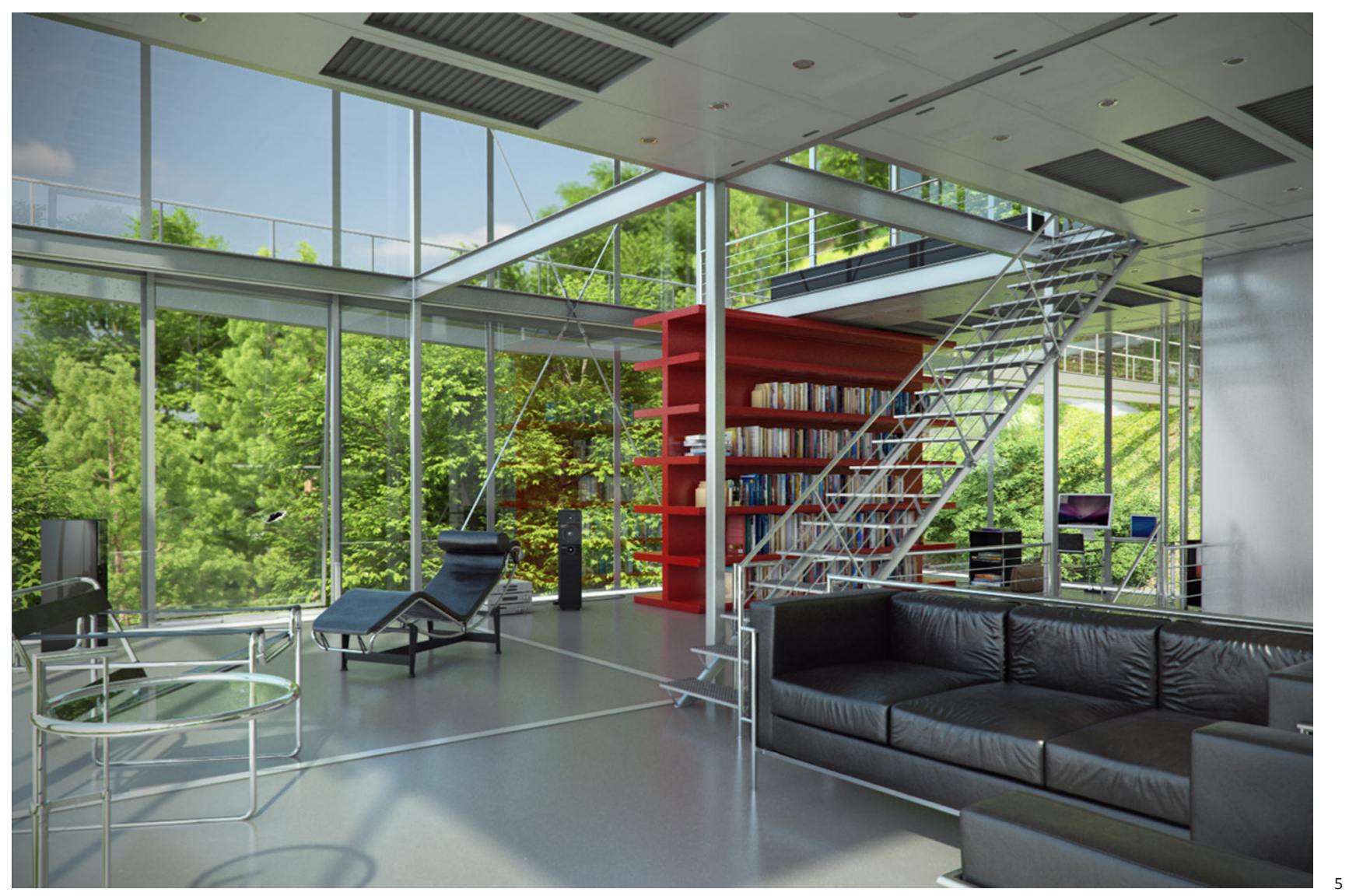

As radical as this sounds, there is a long tradition of building in this way. This practice is millennia old, often associated with temporary structures as seen in the Mongolian yurt or the Blackfoot tipi. However, more permanent structures have utilized this practice such as Paxton's Crystal Palace or more recently, Fuller's American pavilion at the 1967 World's Expo or Sobek's R128 House (Figure 5). The design for re-use and disassembly allows for nimble responses to changing conditions for its users, whether they are hunter/gatherers or millennials. The rapidly changing contemporary context is placing new demands on buildings that require properties of responsiveness and capacity for change while conserving resources. Designing for disassembly (DfD) allows buildings to meet these demands but these new approaches have new social, cultural, and environmental dimensions that the Triakonta system is meant to investigate.

The Triakonta system was designed with these ideas in mind. Not only does it incorporate the low-embodied energy of bamboo into a structure, it offers a modular, reusable structural framing system that is designed to weigh less, sequester carbon, disassemble, and generate exciting new geometries to work with. As the needs of the building change, the building can change without being demolished. At the end-of-life, the metal components can be reused and the natural components can be composted or pyrolized into a soil amendment or a source of cooking fuel. No composites to disentangle, no toxins to dispose of, the materials flows into both the technical and the natural metabolisms that will have to be set up to coexist for us to survive, let alone prosper in the decades to come.
Figure 5: Werner Sobek, R128 House, Stuttgart, 2000 (Photo: Voyager)

7. Dixit, et al. "Identification of parameters for embodied energy measurement: A literature review" Energy and Buildings Energy and Buildings 42 no. 8 (2010): 1238.

8. Ibid., 1239.

9. R.H. Crawford, G.J. Treloar. "Validation of the use of Australian input output data for building embodied energy simulation", Eighth International IBPSA Conference, Eindhoven, Netherlands (2003).

10. "Age of U.S. Commercial Buildings", SMR Research Corporation, Nov. 12, 2009.

11. John Voelcker, "As Gas Mileage Rises, Energy To Make Cars Gets More Important", Green Car Report, 2014.

12. U.S. Environmental Protection Agency, Municipal and Industrial Solid Waste Division, Office of Solid Waste. 1998. "Characterization of BuildingRelated Construction and Demolition Debris in the United States": ES-2.. 